\title{
Success of a sports-club led community X-PERT Diabetes Education Programme
}

\author{
${ }^{\mathrm{a}}$ Morgan, Angela. ${ }^{\mathrm{b}}$ Drew, Dee. ${ }^{\mathrm{c}}$ Clifford, Angela. ${ }^{\mathrm{d}}$ Hull, Katrina. \\ ${ }^{a}$ Centre for Health and Social Care Improvement, Faculty of Education, Health and \\ Wellbeing, University of Wolverhampton, Wolverhampton, United Kingdom. \\ ${ }^{b}$ formerly Centre for Health and Social Care Improvement, Faculty of Education, Health and \\ Wellbeing, University of Wolverhampton, Wolverhampton, United Kingdom. \\ ${ }^{c}$ Centre for Health and Social Care Improvement, Faculty of Education, Health and \\ Wellbeing, University of Wolverhampton, Wolverhampton, United Kingdom. \\ d Public Health, Stoke-on-Trent City Council, Stoke-on-Trent, United Kingdom.
}

Dr Angela Morgan

Centre for Health and Social Care Improvement

Faculty of Education Health and Wellbeing

City campus

University of Wolverhampton

Wolverhampton

WV1 1DT

Email: Angela-Morgan@wlv.ac.uk

Author emails:

Angela Morgan: Angela-Morgan@wlv.ac.uk

Dee Drew: deedrew75@yahoo.com

Angela Clifford: Angela.Clifford@wlv.ac.uk

Katrina Hull: Katrina.Hull@ stoke.gov.uk 


\title{
Success of a sports-club led community X-PERT Diabetes Education Programme
}

\begin{abstract}
This study examined the effectiveness of a sports-club led X-PERT Diabetes Programme, measuring changes to participants' physical condition, knowledge of diabetes, psychological well-being, activity level, diabetes self-management and participants' rating of the programme. In this mixed-method, repeated measures evaluative study, data were gathered at baseline (T0), 6 weeks (T1) and 6 months (T2) using the Diabetes Health Profile (DHP), General Practice Physical Activity Questionnaire (GPPAQ), Diabetes Empowerment Scale - Short Form (DES-SF), Participant Evaluation Questionnaire (PEQ), and focus groups. Over three years, 260 individuals with type 2 diabetes provided data for the evaluation. The sports-club led X-PERT Diabetes Education Programme was successful in achieving its targets with improvement in clinical outcomes including significant reductions in BMI, waist circumference, total cholesterol, LDL cholesterol, triglycerides and glycated haemoglobin by T2. Positive changes were also evident in relation to participants' health profile, self-management and empowerment. This study illustrates the important role that European sports stadia can play in community health through delivering targeted programmes beyond general health promotion activities.
\end{abstract}

Key words: Diabetes; Self-Management; X-PERT Patient; Diabetes Education Programme; Sports Stadia

\section{Introduction}

It is becoming increasingly recognised that sports clubs and their stadia can play an important role in communities and their health behaviours (Healthy Stadia 2015). From being an example of good practice, such as providing healthy catering and having smoking restrictions onsite, to offering open sports facilities for the public, stadia across the UK have pursued an agenda in recent years to improve the health of their fans and the wider community. Some football clubs have also offered targeted community health programmes at their stadia. For 
example, football clubs across the Scottish Premiership League hold Football Fans in Training (FFIT) weight loss and healthy living programmes, funded by the Scottish Government, finding significant reductions in attendees' weight, blood pressure and other clinical outcomes (Hunt et al. 2014).

Sports clubs and their stadia are considered 'icons' in the local area and have unique relationships with the public through their community engagement work, lacking the stigma of a healthcare setting and providing a focus of shared interest (Bunn et al. 2016; Spandler, Roy and McKeown, 2014). They therefore have the potential to support healthcare services in promoting public health and reducing the cost of chronic illness. One such example can be seen in the work of Pringle et al (2014) who assessed the effect of a programme of men's health delivered in/by English Premier League football clubs. In this study, men attended match-day events and/or weekly classes involving physical activity and health education. Men were attracted through football and/or the clubs, and intention-to-treat analysis showed significant improvements in lifestyle profiles. The concept of football clubs delivering health initiatives is relatively recent in comparison to some of the more obvious and established work such as community sports programmes. Nevertheless, the success of such initiatives has paved the way for a constantly evolving health programme at many clubs across the country.

Diabetes is estimated to affect 346 million people worldwide and associated with negative health outcomes and comorbidities including cardiovascular disease, kidney failure and mortality (World Health Organisation 2009), unless the disease is effectively controlled (Skylar et al. 2009). Prevalence of diabetes in the UK was 3.65 million in 2010, with only 2.8 million being aware they have the condition (Diabetes UK n.d.). More than a decade ago, it was estimated that the prevalence of patients diagnosed with diabetes in the UK has doubled in less than 20 years (Harvey, Craney and Kelly 2002). Only one in five people in England and Wales reach targets for keeping their diabetes under control. In England, 19.9\% of people with diabetes meet recommended targets for blood glucose, blood pressure and cholesterol; in Wales, this figure is $18.5 \%$ (Diabetes UK n.d.).

The health of the population of Wolverhampton (currently at approximately 250,000 mid-2012 population estimate (Office of National Statistics n.d.) is worse than the national average, and local prevalence of diabetes reflects national figures (National Health Service 2012; Department of Health 2012). In Wolverhampton, the number of people diagnosed with diabetes in 2010/2011 was 14,846 ; in total $7.1 \%$ of people on GP registers in the town had a recorded diagnosis of diabetes (National Health Service 2012; Department of Health 2012). This was significantly worse than the England average, which for that same year was $5.5 \%$, 
and substantially similar to the indicator proposed in the Public Health Outcomes Framework (Department of Health 2012).

Diabetes health care is an urgent priority and treatment/self-management needs to be improved if a public health disaster is to be avoided (Diabetes UK n.d.). The sharp rise in incidence of type 2 diabetes in the UK is linked to the Westernised lifestyle (Orozco et al. 2008), a term describing the increasingly poor dietary habits and level of physical activity. Current public health initiatives encompass measures to combat obesity, therefore helping to prevent diabetes (Department of Health 2012). Clinical trials have shown that although strengthening glycaemic control can reduce the onset of diabetes-related complications, glycaemic control in diabetic patients has seen no improvement in the last decade (Stratton et al. 2000) whilst prescription costs for diabetes have almost doubled (Currie, Gale and Poole 2010). Controlling blood glucose levels with insulin and sulfonylureas can also lead to serious health challenges attributable to associated weight gain and hypoglycaemia (Fonseca 2003; Amiel et al. 2008; Holman et al. 2009; Duckworth et al. 2009). Evidence clearly demonstrates the need for intervention and education to improve self-management of diabetes. Self-management programmes for diabetes are recognised as the gold standard in treatment (Dineen 2008).

The benefits of self-management programmes are two-fold: they empower patients by giving them tools to manage their condition; and they relieve strain exerted on front-line primary health care practices and hospital emergency departments (Department of Health and Diabetes 2005). The X-PERT Diabetes Education Programme is a self-management programme that aims to help people with type 2 diabetes control many risk factors associated with poor outcomes and to improve their well-being (Department of Health 2001; Deakin et al. 2006). The programme involves group education delivered by trained facilitators, with sessions covering topics such as general awareness, weight management and complications of the disorder. The X-PERT Patient Programme is used by various Clinical Commissioning Groups (CCGs) throughout the UK and, on a national level, has established that it is cost effective, empowering and clinically effective in helping people with type 2 diabetes control many risk factors associated with complications, (X-PERT Health n.d.). However, local evaluation is necessary to ensure that it responds to the needs of individual communities and to share good practice.

\section{Methods}

\section{Intervention context}


The present study describes the implementation and evaluation of the X-PERT Diabetes Education Programme on a local level by Wolves Community Trust, in partnership with the then Wolverhampton Primary Care Trust (PCT) (now the CCG) as part of the Tackle Diabetes initiative. This is one of the Trust's most significant health initiatives. The project was supported by Wolverhampton Wanderers Football Club; the initial project launch was held at Molineux Stadium with WWFC first team players in attendance and as a result attracted local media attention helping to raise the profile of the project. The project also featured on the BBC's Match of the Day's community feature ${ }^{1}$. Wolves Community Trust is a registered charity and an independent organisation. However, it operates as an extension of Wolverhampton Wanderers Football Club, using the brand of the football club, and is based at Molineux Stadium, the home of the football club. The Trust runs a range of health programmes to deliver health education and promote physical activity across all ages. The XPERT programme was delivered as a 3-year local pilot as a practical intervention for diabetes and was delivered at the football stadium and other local settings. The initial project plan included delivery of X-PERT at a number of venues across the city including Molineux Stadium. However, the courses offered at the Stadium were most popular so delivery was predominantly at Molineux with occasional courses delivered in other areas of the city when required. Molineux Stadium is located in a central location of the city with bus stops nearby and free parking was provided for X-PERT participants.

On award of the funding a Project Manager was recruited to deliver the 'Tackle Diabetes' project to develop and implement services to support residents who had type 2 diabetes. Following a mapping exercise, a review of research and recommendation from NICE (CG87 now updated to NG28), the Tackle Diabetes project offered three key components; X-PERT diabetes education programme, Physical Activity and practical Healthy Eating sessions. The Tackle Diabetes project was supported by a full time project administrator employed by the Trust. The role involved processing referrals, arranging courses, dealing with bookings and all other administrative responsibilities for the project. Project plans were discussed and reviewed with the Steering Group which had representation from the local Public Health and Healthy Lifestyle teams, NHS Trust and Wolves Community Trust.

The Diabetes Project Manager and a Dietitian from the local NHS Trust were trained to deliver the X-PERT programme. The training was delivered by X-PERT Health a

\footnotetext{
${ }^{1}$ https://www.youtube.com/watch?v=7zc19t4i2s0
} 
nationally registered charity committed to providing X-PERT Educator courses to healthcare professionals and lay educators to develop their competencies in evidence-based care, enabling them to deliver structured patient education to people with, or at risk of, diabetes (X-PERT Health 2016). Referrals were received from Primary Care, Dietetics and Health Trainers. Self-referrals were also received.

The evaluation of the pilot was conducted by the University and aimed to appraise the effectiveness of a local X-PERT Diabetes Programme in improving participants' physical health, knowledge of diabetes, wellbeing, physical activity and self-management of their diabetes.

\section{Participants}

Participants were recruited via a letter of invitation following a GP referral, Health Care Provider referral, or self-referral. The X-PERT programme was designed specifically for the needs of patients having type 2 diabetes therefore those with type 1 diabetes were excluded. However, so as to be as inclusive as possible, no other exclusion criteria were placed on potential participants. A total of 394 eligible people with type 2 diabetes completed the programme over the 3-year pilot period. Each X-PERT group contained an average of 16 participants, with at least six groups running each year. All groups completed the same programme with no control groups used. During the six week course, participants were given the opportunity to opt in to the programme's evaluative component. Written informed consent was obtained from participants after a full verbal explanation of the evaluation, including identification of any inherent benefits and risks, an explanation of the procedure, and a reminder that their participation in the evaluation was entirely optional and that they may discontinue their participation at any point without explanation and without penalty. The evaluation was reviewed and approved by the University's Faculty of Education, Health and Wellbeing ethics committee.

\section{Design and procedure}

The X-PERT programme consists of six sessions spread over six weeks and the evaluation employed a mixed-method, within-subjects repeated-measures design with three data collection points. Participants who took part in the evaluative component were invited to complete a range of measures pre-programme (T0), post-programme 6-week follow up (T1) and post-programme 6-month follow-up (T2). The Diabetes Health Profile (DHP) was completed at T0 and T2 - this is a validated measure that accurately records an individual's 
medical status (e.g. height, weight, BMI, cholesterol levels, blood pressure, high- and lowlevel lipoprotein, etc.) (Diabetes Profile n.d.). The General Practice Physical Activity Questionnaire (GPPAQ) was also completed at T0 and T2 - this is a tool to assess level of physical activity, including employment, exercise, and walking pace. The Diabetes Empowerment Scale - Short Form (DES-SF) was completed at T0, T1 and T2 - this is a validated tool (Anderson et al. 2003) with eight statements, used to measure participants' level of perceived control over their diabetes over time. The Participant Evaluation Questionnaire (PEQ) was completed at T1 - this is a tool designed to assess the benefit of the programme to participants by rating each week's content and lifestyle experiment (goal setting) on enjoyment, degree of self-management, impact on health, and impact on living with diabetes (World Health Organisation 2009). Comments were also invited on the venue, length of programme, most enjoyable or useful weeks, and most unhelpful weeks.

Two focus groups (Wilkinson 1998) were held at T1 with participants to capture more nuanced qualitative data usually difficult to obtain with questionnaire-based measures. The first focus group comprised six participants, the second included six participants and one carer. Discussions promoted open and frank conversations of issues that participants felt were of particular importance to them during the programme. They provided a wealth of qualitative data with which to analyse participants' perceptions of the programme. In addition, the discussions acted as a method of evaluating the links the programme has made with various support services across the region, determining the utility of their inclusion within the programme, including local Healthy Lifestyle, Dieticians and Healthy Minds services.

\section{Data analysis}

Quantitative data were analysed using the Statistical Package for Social Sciences (SPSS) v16. A range of descriptive and comparative statistical techniques used a within-subjects repeated measures design (t-test and Analysis of Variance [ANOVA] for continuous data, Wilcoxon Signed Rank Test for ordinal data), wherein participants' baseline and follow-up measures (pre- and post-programme) were compared. An intention-to-treat analysis was used in which all participants were included in the final analysis even if they missed more than one session of the programme. Qualitative data obtained from the focus groups and PEQ were analysed using thematic analysis, a technique that focuses upon understanding and interpreting informants' experiences of any given issues (Braun and Clarke 2006). This dataset was analysed by two researchers without the use of computer-assisted qualitative data analysis 
software due to the relatively small sample size. Initial basic coding procedures were followed by more complex interpretive analysis and corroboration of themes by the two analysts.

\section{Results}

Of 394 participants who completed the X-PERT program during the study period, 260 agreed to take part in the evaluative component. Of those, 252 (99\%) participants attended four or more sessions and $150(58 \%)$ attended all six. A total of 296 participants provided data for analysis. Table 1 shows the gender, age and ethnicity of participants. Black and Asian groups were underrepresented but there was an even split for gender. Median age fell within the 6170 year age group for men and the 51-60 year age group for women, with the age distribution skewed towards the middle- to older-age groups.

Table 1: Demographic information of participants

\begin{tabular}{lll}
\hline & $N$ & $\%^{a}$ \\
\hline Mender & 119 & \\
Male & 160 & 42.7 \\
Female & 1 & 57.3 \\
\hline Age & 10 & 0.3 \\
$20-30$ & 42 & 3.5 \\
$31-40$ & 88 & 14.7 \\
$41-50$ & 96 & 30.9 \\
$51-60$ & 45 & 33.7 \\
$61-70$ & 3 & 15.8 \\
$71-80$ & & 1.0 \\
$81-90$ & 74 & 25.3 \\
Ethnicity & 20 & 6.8 \\
Asian & 197 & 67.5 \\
Black & 1 & 0.3 \\
White & & \\
Mixed ethnicity & &
\end{tabular}

\section{Changes in physical activity}

Scores on the General Practice Physical Activity Questionnaire (GPPAQ) showed that, at T0, nearly half of participants (46.2\%) scored as 'inactive' while 19.8\% scored as 'active'. 11.5\% of participants spent 3 hours or more per week in physical exercise (e.g. swimming, jogging, aerobics, football, tennis, gym) and $20.5 \%$ spent 1 hour or more per week in physical 
exercise. Nearly half (46.7\%) spent one hour or more per week walking, though walking pace was mostly slow or steady (79.7\% of participants). Wilcoxon Signed Ranks Tests (for ordinal data) found no statistically significant differences in activity levels $(Z=-1.446, p=.148)$, time spent in exercise per week $(\mathrm{Z}=0.148, \mathrm{p}=.879)$, time spent walking $(\mathrm{Z}=-0.170, \mathrm{p}=.865)$ or walking pace $(\mathrm{Z}=-0.626, \mathrm{p}=.532)$ between $\mathrm{T} 0$ and $\mathrm{T} 2$ (missing data meant that $<86$ participants were included in each comparison).

\section{Physiological changes}

Table 2 shows physiological measurements at baseline and follow-up, compared using paired-samples t-tests (due to missing data there was insufficient power to conduct an ANOVA). All measurements except HDL cholesterol showed modest movement in the desired direction between T0 and T2 and this change was statistically significant for waist circumference, BMI, total cholesterol, LDL cholesterol, Triglycerides and HbA1c. After adjusting the alpha value for multiple comparisons using the Holm-Bonferroni method, observed changes in BMI, total cholesterol, LDL and HbA1c remained statistically significant.

Table 2: Changes in key physiological measurements from T0 to T2

\begin{tabular}{llllll}
\hline Measurement & \multicolumn{2}{c}{ T0 } & \multicolumn{2}{c}{ T2 } & \multirow{2}{*}{$t$} \\
\cline { 2 - 5 } & Mean & SD & Mean & SD & 0.36 \\
Weight $(\mathrm{kg})$ & 88.2 & 22.7 & 87.5 & 20.0 & $2.38^{*}$ \\
Waist $(\mathrm{cm})$ & 103.2 & 16.8 & 95.4 & 22.0 & $3.33^{*}$ \\
BMI & 32.0 & 6.5 & 31.1 & 6.2 & $2.87^{*}$ \\
Total cholesterol & 4.7 & 1.3 & 4.3 & 1.1 & -0.82 \\
HDL & 1.4 & 0.5 & 1.4 & 0.9 & $2.90^{*}$ \\
LDL & 2.8 & 1.2 & 2.5 & 1.1 & $2.54^{*}$ \\
Triglycerides & 1.7 & 1.0 & 1.4 & 0.8 & $3.33^{*}$ \\
HbA1c $(\%)$ & 9.8 & 1.5 & 8.3 & 1.2 &
\end{tabular}

$* \mathrm{p} \leq .05$

\section{Use of medication}

Of 47 participants providing data regarding medication use, most remained stable during the course of the program. Two participants reported a decrease in medication and four reported an increase in medication use. A further two participants who had not previously taken medication for their diabetes started doing so during the program. The reasons for alterations in medicinal treatment were undetermined. 


\section{Diabetes Empowerment Scale - Short Form (DES-SF)}

Mean scores on the DES-SF at T0, T1 and T2 increased between baseline and postintervention (Figure 1). Repeated Measures ANOVA showed that this increase was statistically significant $(\mathrm{F}(2,170)=40.00, \mathrm{p}=.000)$. The small drop-off between $\mathrm{T} 1$ and $\mathrm{T} 2$ was not statistically significant and may reflect the small number of participants at T2 $(n=63)$.

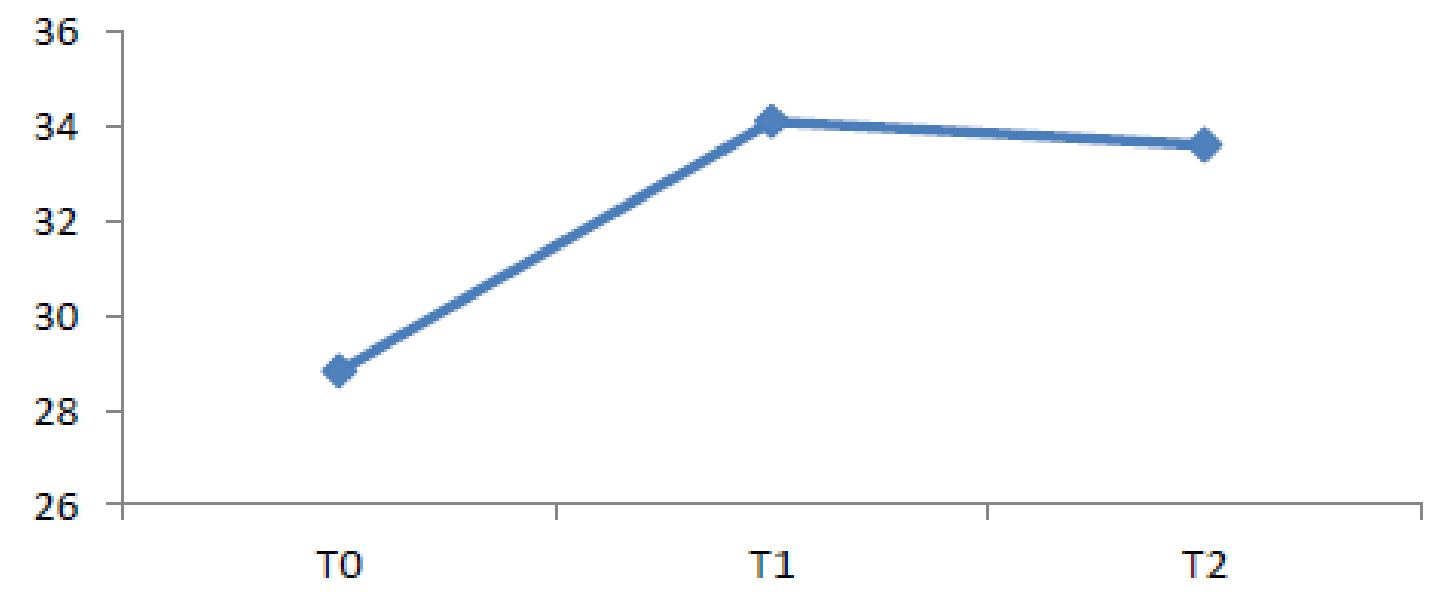

Figure 1: Scores on the DES-SF at T0, T1 and T2

\section{Participant Evaluation Questionnaire (PEQ)}

212 participants completed the PEQ at T1 covering the topics from weeks 1-6. Responses are described here briefly. Over $92 \%$ of participants responded favourably to the weekly topics being 'really enjoyable and really useful'; 'will improve their self-management skills'; 'will greatly improve their health'; and 'will help them to live with diabetes'. Comments on all weekly topics were positive and participants stated how much they valued the programme, organisation, venue, group dynamics, and personal skills of the Educators. Most participants thought that the lifestyle experiment was 'really enjoyable and really useful' (92\%), will 'improve self-management skills' (88\%), will 'greatly improve their health' (85\%), and will 'help them to live with diabetes' (84\%).

$179(84 \%)$ respondents stated that they found the diabetes handbook 'really useful' and $175(82 \%)$ stated that the handbook will help a lot with their self-management of diabetes. Overall it was felt by participants that the handbook on diabetes is an excellent reference resource both during and after the programme, but one participant reported that the handbook could be improved by using larger font for those with a visual impairment. 161 
(82\%) participants stated that the length of the programme was just right, though some participants indicated that a refresher course would be valued. Some recommendations were made regarding accessibility and comfort of the venue.

\section{Focus group data}

A number of themes were generated through the focus groups. The key reason for attending the course was that it was considered the best place to obtain specialist information that was not available elsewhere. For example, one participant stated,

Well, when I was diagnosed, the doctor told me nothing. She just told me 'you're borderline diabetic'. I've learned since that there is no such thing as 'borderline', so for the past six years I really didn't know what to do.

General experiences of the course were entirely positive, with some participants stating that they are now recommending the course to others who have diabetes. The delivery of the programme was said to be interesting and effective for participants, with many participants praising the Educator,

Just as I said before, in just two words, [The Educator]. Because she is the person who puts it over in the way we can understand, she isn't regimental in the programme step-by-step. You can ask her anything, she'll give you the answer, she'll give you advice and you gain confidence to go back to the people who you need to speak to in the surgery and query what's happening... But definitely, [The Educator] ... because all the people that have not been diagnosed or coming up to being diagnosed need to come through her programme.

Key lessons learned included myth-busting as a way of changing to a healthier lifestyle. Acceptance was a major theme to develop with the focus group discussions, with many participants stating that they had at first not entirely believed their diagnosis of diabetes and had gone into a state of denial. The information presented reduced and allayed their fears about their current and future health.

Positively having and maintaining control over their diabetes was another major theme to emerge from the data, especially in terms of dietary self-control and medication side-effects. One participant stated, 
They [doctors] give you tablets but rarely tell you how it operates. On this course, you can find out what they do and it helps you to deal with it.

The involvement of a local sports club in its support and sharing resources was particularly valued by participants; being aligned with the legendary and celebrated nature of the club has its own rewards for some participants. One participant reported that it felt special to be at the [sports] complex' and others stated,

I thought it was rather nice when [footballer] came... and then we had a day session; some of the footballers, they came and they were doing the dance with us, we were doing the Zumba.

When I spoke to [footballer] he said he had a glass of water every morning to clear his system out, and I have done that ever since!

This view on the importance of the programme being held at a sports stadium reflects those of previous studies (Bingham, Richardson, Curran, and Parnell 2014; Curran, Bingham, Richardson and Parnell 2014).

\section{Discussion}

This study identified positive outcomes from the sports-club led X-PERT Diabetes Education Programme in terms of participants' physiological and psychological factors as well as satisfaction with the programme. The programme was well attended and impacted positively upon participants' confidence and self-empowerment. Participants considered the programme to be a valuable source of information, guidance and support for maintaining control of one's own diabetes that was widely felt was not available elsewhere. Significant reductions were observed in some, though not all, risk factors for diabetes complications (e.g. waist circumference, BMI, total and LDL cholesterol, triglycerides and blood glucose), which may be due to the relatively small sample size and short timescale. Participants were generally inactive at baseline, but physical activity levels did not change over the course of the programme. However, with physical activity being a key intervention for preventing or delaying the onset of type 2 diabetes (World Health Organisation 2009), further considerations for others working in this area are that football clubs and their stadia might play an additional and important role in addressing this part of diabetes self-management in the future through their general sports facilities. 
These outcomes and indicators of effectiveness of the X-PERT programme appear to be comparable to those shown nationally (Deakin et al. 2006) though a direct comparison in clinical outcomes is difficult due to different follow-up timescales and baseline measurements. National data on 16,919 participants has shown the effectiveness of the XPERT Patient Programme in many areas. For example, a very high total participant evaluation score at 94\%; an increase in empowerment score by $23 \%$ post-programme and $26 \%$ at one-year follow-up; reduction in glycated haemoglobin (HbA1c), weight and waist circumference at six months and 14-month follow-up; and reduction in both systolic and diastolic blood pressure and lipids (Deakin et al. 2006). Compared to $82 \%$ in the national evaluation who attended four or more sessions, $99 \%$ of participants in the present study attended four or more sessions. Although only $4 \%$ of participants in the present study showed reduction in medication use compared to $16 \%$ in the national evaluation, this was over a much shorter time span and data were based on a low response rate. This comparability between local and national outcomes suggests that sports clubs can be at least as effective as other healthcare providers in delivering structured health education.

The location of the programme within Wolverhampton Wanderers Football Club was extremely important and had a positive impact on participants. This was reflected in their comments during the focus groups which highlighted how the location within a prominent and important local venue impacted on their sense of pride and belonging and may have explained the higher rate of attendance in comparison to the national evaluation. These findings concur with Pringle et al (2014). This reflects the importance of the football club as a symbol of the city, and it was further reinforced to participants when players from the football team joined them on the programme. Previous studies such as FITT (Hunt et al. 2014) and an Improving Access to Psychological Therapies (IAPT) funded mental health project in North West England (Spandler, McKeown, Roy and Hurley 2013) have shown similar benefits of utilising sporting venues as health intervention settings with positive clinical outcomes and service user feedback. This emphasises the importance of utilising spaces and possibly engaging public figures, which have resonance with the local target population. This should encourage service providers to consider popular venues which may not be immediately identified as 'health centres' but which have a currency with the groups they are aiming to target.

Wolverhampton CCG identified 14,000 patients diagnosed with diabetes living in the city, and the programme has the potential to be offered to all patients through the practitioner 
referral system. Annual inpatient care to treat short- and long-term complications of diabetes is estimated at between $£ 1,800$ and $£ 2,500$ per patient (Diabetes UK n.d.), in contrast to annual outpatient costs (including costs of medications and monitoring supplies) estimated at between $£ 300$ and $£ 370$ per patient. Crude estimates of costs savings from this cohort suggest that if the need for inpatient care has reduced in $50 \%$ of the sample due to reduction or stabilisation of medication $(n=130)$, there is a potentially large saving each year from this sample alone. In addition to the health economic considerations, the findings also highlight other opportunities relating to the planning and delivery of community based interventions to reduce diabetic risk and optimise self-agency and self-care among people living with diabetes. As a short intervention (6 weeks) the X-PERT programme shows significant economic and social cost savings, although the effectiveness of the programme in maintaining these behaviours long term requires further evaluation.

Some limitations of the current study were identified that should be addressed in future work around the X-PERT Diabetes Education programme, whether led by sports clubs or by other providers. Young people and some ethnic groups were underrepresented in this particular cohort; the majority were aged 51-70 years and less than one third were from BME populations. Further evaluation is needed to ensure that the positive outcomes are observed in these groups and further iterations of the programme should aim to reach a diverse audience, especially since participants were effectively self-selected. Targeting younger people (those diagnosed when under the age of 25) with diabetes often referred to as having 'maturity onset diabetes of the young' (MODY) for the X-PERT programme will also give them the opportunity to learn about self-management sooner following diagnosis, thus maximising the health and wellbeing benefits of the programme. However, MODY is different to type 1 and type 2 diabetes and thus young patients will need specialist advice and care within such a self-management programme.

Some participants reported that they found out about the programme themselves and had to request a referral from their GP. A clear and robust referral process including education and information for primary care practitioners and diabetes specialist nurses as to the programme on offer is thus recommended to reach as many people as possible. Advertising of the programme could also be maximised through sport clubs' own community engagement channels, given their visible presence in the local area. One practical limitation of evaluative work of a non-NHS provider was that the programme organisers reported difficulty obtaining participant data directly, instead relying upon participants to bring that 
data to them. This resulted in fewer data at follow-up compared to baseline and represents an ongoing challenge for collecting data from non-NHS services.

\section{Conclusion}

In conclusion, this study has illustrated an effective partnership between a sports club and its stadium and a healthcare provider to improve the health of the community, using an existing targeted programme with measureable benefits. The delivery of this programme by sports clubs elsewhere could play an important role in the care of people living with type 2 diabetes locally by reducing risk factors for complications without the need for pharmacological interventions, and in doing so relieving pressure upon primary care services.

\section{Acknowledgements}

The authors wish to thank Wolves Community Trust and all the individuals who gave of their time to support and contribute to this work.

\section{Disclosure Statement}

No potential conflict of interest was reported by the authors.

\section{Funding}

Wolves Community Trust

\section{References}

Amiel, S.A., T. Dixon, R. Mann and K. Jameson. 2008. Hypoglycaemia in Type 2 diabetes. Diabetic Medicine 25 (3): 245-254. doi: 10.1111/j.14645491.2007.02341.x

Anderson, R.M., J.T. Fitzgerald, L.D. Gruppen, M.M. Funnell and M.S. Oh. 2003. The Diabetes Empowerment Scale-Short Form (DES-SF). Diabetes Care 26 (5): 1641-2. doi: 10.2337/diacare.26.5.1641-a 
Bingham, D.D., D. Richardson, K. Curran and D. Parnell. 2014. Fit Fans: perspectives of a practitioner and understanding participant health needs within a health promotion programme for older men delivered within an English Premier League Football Club. Soccer and Society, 15 (6), 883-901.

Braun, V., and V. Clarke. 2006. Using thematic analysis in psychology. Qualitative Research in Psychology 3 (2): 77-101. doi: 10.1191/1478088706qp063oa

Bunn, C., S. Wyke, C.M. Gray, A. Maclean and K. Hunt. 2016. 'Coz football is what we all have': masculinities, practice, performance and effervescence in a gender- sensitised weight-loss and healthy living programme for men. Sociol Health Illn. doi: 10.1111/1467-9566.12402. [Epub ahead of print]

Curran, K., D.D. Bingham, D. Richardson, and D Parnell. 2014. Ethnographic engagement from within a Football in the Community programme at an English Premier League football club. Soccer and Society, 15 (6), 934-950.

Currie, C.J., E.A. Gale and C.D. Poole. 2010. Estimation of primary care treatment costs and treatment efficacy for people with type 1 and type 2 diabetes in the United Kingdom from 1997 to 2007. Diabetic Medicine 27 (8): 938-48. doi: 10.1111/j.14645491.2010.03040.x

Deakin, T.A., J.E. Cade, R. Williams and D.C. Greenwood. 2006. Structured patient education: the Diabetes X-PERT Programme makes a difference. Diabetic Medicine 23: 944-54. doi: 10.1111/j.1464-5491.2006.01906.x

Department of Health. 2001. The Expert Patient: A New Approach to Chronic Disease Management in the 21st Century. London: The Stationery Office.

Department of Health. 2012. Improving outcomes and supporting transparency: Part 1: A public health outcomes framework for England, 2013-2016. Accessed 6 Jan 2014.https://www.rcpsych.ac.uk/pdf/The\%20Public\%20Health\%20Outcomes\%20Fra mework.pdf

Department of Health and Diabetes UK. 2005. Structured Patient Education in Diabetes: Report from the Patient Education Working Group. London: Crown COI for the Department of Health. 
Diabetes Profile (n.d.) Accessed 1 Aug 2014. http://www.diabetesprofile.com/

Diabetes UK (n.d.). Cost of Diabetes. Accessed 20 Dec 2013. http://www.diabetes.co.uk/costof-diabetes.html

Dinneen, S.F. 2008. Structured education for people with type 2 diabetes. British Medical Journal 336 (7642): 459-60. doi: 10.1136/bmj.39478.693715.80

Duckworth, W., C. Abraira, T. Moritz, D. Reda, N. Emanuele, P.D. Reaven, et al. 2009. Glucose control and vascular complications in veterans with type 2 diabetes. New England Journal of Medicine 360 (2): 129-39. doi: 10.1056/NEJMoa0808431

Fonseca, V. 2003. Effect of thiazolidinediones on body weight in patients with diabetes mellitus. American Journal of Medicine 115 (Suppl 8A): 42-8. Doi: 10.1016/j.amjmed.2003.09.005

Harvey, J.N., L. Craney and D. Kelly. 2002. Estimation of the prevalence of diagnosed diabetes form primary care and secondary care source data: comparison of record linkage with capture-recapture analysis. Journal of Epidemiology and Community Health 56: 18-23. doi: 10.1136/jech.56.1.18

Healthy Stadia. 2015. The Healthy Stadia® concept. Accessed 14 September 2015. http://www.healthystadia.eu/about.html

Holman, R.R., A.J. Farmer, M.J. Davies, J.C. Levy, J.L. Darbyshire, J.F. Keenan, et al. 2009. Three-year efficacy of complex insulin regimens in type 2 diabetes. New England Journal of Medicine 361 (21): 1736-47. doi: 10.1056/NEJMoa0905479

Hunt, K., S. Wyke, C.M. Gray, A.S. Anderson, A. Brady, C. Bunn, P.T. Donnan, et al. 2014. A gender-sensitised weight loss and healthy living programme for overweight and obese men delivered by Scottish Premier League football clubs (FFIT): a pragmatic randomised controlled trial. Lancet. 383: 1211-21. doi: 10.1016/S01406736(13)62420-4

National Health Service (n.d.). Health profile 2012: Wolverhampton. Accessed 14 Dec 2013. www.healthprofiles.info

Office for National Statistics. (n.d.) Accessed 14 Dec 2013. www.ons.gov.uk 
Orozco, L.J., A.M. Buchleitner, G. Gimenez-Perez, M.R. Figuls, B. Richter and D. Mauricio. 2008. Exercise or exercise and diet for preventing type 2 diabetes mellitus (Review). The Cochrane Collaboration 3 (2): CD003054. doi: 10.1002/14651858.CD003054.pub3

Pringle, A, Zwolinsky, S, McKenna, J, Robertson, S, Daly-Smith, A and White, A. (2014). "Health Improvement for Men and Hard-to-Engage Men Delivered in English Premier League Football Clubs." Health Education Research 29 (3):503-520.

Spandler, H., M. Mckeown, A. Roy, M.J. Hurley. 2013. Football metaphor and mental well-being: an evaluation of the It's a Goal! programme. Ment Health 22 (6): 544-54. doi: 10.3109/09638237.2013.819420

Spandler, H., A. Roy and M. Mckeown. 2014. Playing by the Rules? Gender Relations in a Football and Mental Health Project. The Journal of Men's Studies 22 (2): 140-154. doi: $10.3149 /$ jms. 2202.140

Stratton, I.M., A.I. Adler, H.A. Neil, D.R. Matthews, S.E. Manley, C.A. Cull, et al. 2000. Association of glycaemia with macrovascular and microvascular complications of type 2 diabetes (UKPDS 35): prospective observational study. British Medical Journal 321 (7258): 405-12. doi: 10.1136/bmj.321.7258.405

Wilkinson, S. 1998. Focus Groups in Health Research Exploring the Meanings of Health and Illness. Journal of Health Psychology 3 (3): 329-48. doi: $10.1177 / 135910539800300304$

World Health Organisation. 2009. WHO Diabetes. Accessed 2 Nov 2010. http://www.who.int/mediacentre/factsheets/fs312/en/

X-PERT Health. (n.d.). Accessed 1 Aug 2014. http://www.xperthealth.org.uk/ 